\title{
Controlling Nutritional Status (CONUT) Score Is a Prognostic Marker in Metastatic Colorectal Cancer Patients Receiving First-line Chemotherapy
}

\author{
NOBUYA DAITOKU, YUJI MIYAMOTO, RYUMA TOKUNAGA, YUKI SAKAMOTO, YUKIHARU HIYOSHI, \\ MASAAKI IWATSUKI, YOSHIFUMI BABA, SHIRO IWAGAMI, NAOYA YOSHIDA and HIDEO BABA \\ Department of Gastroenterological Surgery, \\ Graduate School of Medical Sciences, Kumamoto University, Kumamoto, Japan
}

\begin{abstract}
Background/Aim: The Controlling Nutritional Status (CONUT) score is a useful nutritional evaluation, that is calculated from serum albumin, total cholesterol concentrations, and total lymphocyte count. This study aimed to investigate the association between the CONUT score and prognosis in patients with metastatic colorectal cancer $(m C R C)$. Patients and Methods: The CONUT score was retrospectively calculated in 211 patients with $m C R C$ receiving first-line chemotherapy. The patients were divided into three groups: the CONUT low-group (0-1), intermediate-group (24), and high-group (5-). The associations of the CONUT score with clinicopathological factors and survival outcomes were evaluated. Results: The higher CONUT score was significantly associated with synchronous metastases, and no primary tumor resection. The higher CONUT score group showed a significant shorter progression-free survival (log-rank $p<0.05)$ and overall survival (log-rank $p<0.001)$. Conclusion: The CONUT score is a useful prognostic marker for predicting survival outcomes of patients with $\mathrm{mCRC}$.
\end{abstract}

Colorectal cancer (CRC) is the third most commonly diagnosed cancer and the second leading cause of cancerrelated deaths worldwide (1). Over the past decades, treatment outcomes for metastatic CRC (mCRC) patients have improved remarkably (2). This has been driven to a large extent by the approval of new drugs, including irinotecan, oxaliplatin, capecitabine, several humanized monoclonal antibodies, and most recently, regorafenib and trifluridine/tipiracil (TAS-102)

Correspondence to: Dr. Hideo Baba, Department of Gastroenterological Surgery, Graduate School of Medical Sciences, Kumamoto University, 1-1-1 Honjo, Kumamoto 860-8556, Japan. Tel: +81 963735212, Fax: +81963714378, e-mail: hdobaba@kumamoto-u.ac.jp

Key Words: CONUT score, metastasis, colorectal cancer, prognostic marker.
$(3,4)$. The clinical benefit from these drugs is now well established for mCRC patients, with the median overall survival (OS) increasing to over 30 months (2); however, more reliable and specific biomarkers are needed.

Patients' nutritional status has recently been associated with prognosis in mCRC (5). The nutritional condition of mCRC patients is an important factor because it allows an estimation of treatment tolerability and cancer progression. A poor nutritional condition is reported to be associated with tumor progression (6), and may reflect elevated metabolism caused by the cancer, an immunocompromised status due to tumor progression, or treatment intolerance (7). A systemic immunological condition is also reported to be associated with cancer prognosis (8). Blood neutrophil, lymphocyte, monocyte, and platelet counts are reported to reflect systemic and local inflammation associated with cancer progression and prognosis (7).

Controlling Nutritional Status (CONUT) score is a new scoring system for patients' nutritional status (9), similar to the modified Glasgow Prognostic Score (mGPS) and the neutrophil-to-lymphocyte ratio (NLR) (10). CONUT score is easily calculated and has been reported to be a predictor for postoperative gastrointestinal cancers (11-15), liver disease (1618) and heart failure (19-21). CONUT score is calculated from three clinical parameters: serum albumin, total cholesterol, and total lymphocyte count. These are readily obtained parameters from routine blood examinations during hospital stays. Previously, we reported that CONUT score may predict survival and severe complications after curative CRC surgery (15). However, associations between CONUT score and mCRC patient outcomes have not been examined. Therefore, this study aimed to evaluate the efficacy of CONUT score for predicting the outcomes of $\mathrm{mCRC}$ patients.

\section{Patients and Methods}

Patients and CONUT scoring. We retrospectively assessed 211 consecutive mCRC patients who received systemic first-line chemotherapy between January 2005 and March 2014 at Kumamoto 
University (Kumamoto, Japan). The eligibility criteria included histologically confirmed CRC and measurable metastatic disease according to Response Evaluation Criteria in Solid Tumors (RECIST), and no previous exposure to systemic chemotherapy for metastatic disease. The exclusion criteria included patients who lacked full blood counts or serum albumin and/or total cholesterol levels measured one month before first-line chemotherapy. We collected the following data from inpatient and outpatient records for clinical characteristics: sex, age, body mass index (BMI), carcinoembryonic antigen (CEA) levels (ng/ml), CA19-9 levels $(\mathrm{U} / \mathrm{ml})$, metastasis (synchronous or metachronous, single or multiple), liver metastasis (only or not only), primary tumor location (right-side or left-side), whether the primary was resected or not, and KRAS status. BMI was calculated from the pretreatment patient heights and weights, which were measured by our medical staff a few days before chemotherapy began. All patients were evaluated for progression-free survival (PFS) and OS.

In this study, CONUT score was used as an indicator of nutritional status and prognosis. Serum albumin and total cholesterol levels, and total lymphocyte count were measured to calculate CONUT score (Table I). All patient blood samples were obtained within one month before they received first-line chemotherapy. Patients were divided into three groups according to their CONUT score: the low score (0-1), intermediate score (2-4), and high score (5-) groups. The use of clinical data in this study was approved by the human ethics review committee of the Graduate School of Medicine, Kumamoto University and the Helsinki Declaration of 1964. Informed consent was obtained from all patients before they were included in this study. This study was conducted in adherence to the REporting recommendations for tumor MARKer prognostic studies (REMARK) (22).

Statistical analyses. All statistical analyses were performed using JMP (version 13; SAS Institute, Cary, NC, USA). The log-rank test was used to determine statistical differences between groups. Cox proportional hazard analyses were performed to determine prognostic factors. A $p$-value of less than 0.05 was considered significant. Univariate analyses were performed to investigate the correlation between CONUT score and clinicopathological factors. Categorical variables were analyzed by the chi-square test or Fisher's exact test, and continuous variables were analyzed by Student's $t$-test. The Kaplan-Meier method and log-rank test were used for survival analyses. Cox proportional hazards regression models were used to calculate hazard ratios (HRs) and 95\% confidence intervals (CIs).

\section{Results}

Correlations between CONUT scores and clinicopathological factors. Baseline characteristics of the patients are shown in Table II. Among the 211 CRC patients included in this study, $126(59.7 \%)$ were male and $85(40.3 \%)$ were female; their median age was 63.0 years (range $=34-86$ years). The patients were divided into three groups: patients with a CONUT score of 1 or less were defined as the CONUT-low group, patients with a score of 2-4 were defined as the CONUT-intermediate group, and those with a score of 5 or more were defined as the CONUT-high group for analyses of correlations with clinicopathological factors (Table III). CONUT score was
Table I. Definition of CONUT score.

\begin{tabular}{lcccc}
\hline Parameters & Normal & Light & Moderate & Severe \\
\hline Serum albumin $(\mathrm{g} / \mathrm{dl})$ & $\geq 3.5$ & $3.0-3.49$ & $2.5-2.9$ & $<2.5$ \\
score & 0 & 2 & 4 & 6 \\
Total lymphocyte $\left(\right.$ count $\left./ \mathrm{mm}^{3}\right)$ & $\geq 1600$ & $1200-1599$ & $800-1199$ & $<800$ \\
score & 0 & 1 & 2 & 3 \\
Total cholesterol $(\mathrm{mg} / \mathrm{dl})$ & $\geq 180$ & $140-180$ & $100-139$ & $<100$ \\
score & 0 & 1 & 2 & 3 \\
CONUT score (total) & $0-1$ & $2-4$ & $5-8$ & $9-12$ \\
Assessment & Low & Intermediate & \multicolumn{2}{c}{ High } \\
\hline
\end{tabular}

CONUT: Controlling nutritional status.

Table II. Baseline characteristics of the patients.

\begin{tabular}{lc}
\hline & $\mathrm{n}=211$ \\
\hline Age, years (range) & $63.0(34-86)$ \\
Male/Female & $126 / 85$ \\
Body mass index, $\mathrm{kg} / \mathrm{m}^{2}$ (range) & $22.8(15.3-33.2)$ \\
Time to metastases, synchronous/metachronous & $162 / 49$ \\
Number of metastases, single/multiple & $103 / 108$ \\
Liver only metastases, yes/no & $80 / 131$ \\
Primary tumor location, right-side/left-side & $60 / 151$ \\
Primary tumor resection, yes/no/unknown & $95 / 107 / 9$ \\
KRAS status, wild type/mutant/unknown & $101 / 53 / 57$ \\
CEA, ng/ml (range) & $497.9(0.5-18021)$ \\
CA19-9, U/ml (range) & $1587.0(0.1-67200)$ \\
\hline
\end{tabular}

CEA: Carcinoembryonic antigen; CA19-9: carbohydrate antigen 19-9.

significantly associated with several clinicopathological factors; metastasis (synchronous or metachronous, $p<0.001$ ) and primary tumor resection $(p<0.001)$ were significantly lower in the CONUT-high group than in the CONUT-low group. There were no other significant differences between the three groups regarding these clinical parameters.

Correlations between CONUT score and survival outcomes. Figure 1 shows the Kaplan-Meier analysis for OS and PFS according to CONUT score. The median 5-year OS and PFS were 24.5 and 6.9 months. Five-year OS rates in the low, intermediate, and high groups were $22.4 \%, 21.4 \%$, and $9.1 \%$, respectively $(p<0.001)$. Both OS and PFS survival curves were better in patients with low or intermediate status than for those with CONUT-high status (OS; $p<0.001, \mathrm{PFS} ; p<0.05$ ). In Cox hazard analyses, univariate analysis showed that CA19-9 concentration ( $>37.0 \mathrm{U} / \mathrm{ml}, p<0.05$ ), metastasis (synchronous, $p<0.05$ ), metastatic lesions (multiple, $p<0.05$ ), liver metastasis (not only, $p<0.05$ ), primary tumor location (right-side, $p<0.001$ ), primary resection (no, $p<0.05$ ), and CONUT score (high, $p<0.001$ ) were significantly associated with worse OS 
Table III. Clinical characteristics of the three groups according to the CONUT score.

\begin{tabular}{|c|c|c|c|c|}
\hline & Low $(n=89)$ & Intermediate $(\mathrm{n}=90)$ & High $(n=32)$ & $p$-Value \\
\hline Male/Female & $58 / 31$ & $51 / 39$ & $17 / 15$ & 0.30 \\
\hline Age, years (range) & $63.1(35-85)$ & $62.3(34-81)$ & $64.5(49-86)$ & 0.57 \\
\hline Body mass index, $\mathrm{kg} / \mathrm{m}^{2}$ (range) & $23.8(16.8-32.5)$ & $22.0(15.9-33.2)$ & $22.2(15.3-29.5)$ & 0.20 \\
\hline CEA, ng/ml (range) & $281.8(0.5-6319)$ & $468.0(0.6-11210)$ & $1184.9(1.8-18021)$ & 0.25 \\
\hline CA19-9 (U/ml), range & $1256.8(0.1-67200)$ & $1019.9(0.1-30860)$ & $4082.6(0.6-47050)$ & 0.16 \\
\hline Metastasis, synchronous/metachronous & $64 / 25$ & $66 / 24$ & $32 / 0$ & $<0.001$ \\
\hline Metastatic lesions, single/multiple & $48 / 41$ & $43 / 47$ & $12 / 20$ & 0.27 \\
\hline Liver metastasis, only/not only & $39 / 50$ & $34 / 56$ & $7 / 25$ & 0.08 \\
\hline Primary tumor location, right-side/left-side & $22 / 67$ & $29 / 61$ & $9 / 23$ & 0.54 \\
\hline Primary tumor resection, yes/no/unknown & $45 / 40 / 4$ & $48 / 38 / 4$ & $2 / 29 / 1$ & $<0.001$ \\
\hline KRAS status, wild type/mutant/unknown & $42 / 20 / 27$ & $41 / 22 / 27$ & $18 / 11 / 3$ & 0.14 \\
\hline White blood cell count, $/ \mu 1$ (range) & $6742.7(3100-14400)$ & $6381.1(2200-26600)$ & $8459.4(2900-19800)$ & 0.08 \\
\hline Total lymphocyte count, / $\mu \mathrm{l}$ (range) & $1878.5(1210-4423)$ & $1416.0(445-3382.5)$ & $1258.1(509-3524)$ & $<0.001$ \\
\hline Albumin, g/dl (range) & $4.0(3.5-4.8)$ & $3.75(3.0-4.9)$ & $2.6(1.8-3.1)$ & $<0.001$ \\
\hline Total cholesterol, $\mathrm{mg} / \mathrm{dl}$ (range) & $207.2(142-391)$ & $191.8(116-397)$ & $190.4(111-350)$ & 0.04 \\
\hline
\end{tabular}

Low: Low CONUT score (0-1) group; Intermediate: intermediate CONUT score (2-4) group; High: high CONUT score (5-) group; CEA: carcinoembryonic antigen; CA19-9: carbohydrate antigen 19-9.

(Table IV). Multivariate analysis showed that CONUT score was an independent prognostic factor for OS (high vs. intermediate/low, $\mathrm{HR}=2.01,95 \% \mathrm{CI}=1.26-3.12, p<0.05)$.

\section{Discussion}

CONUT score is a nutritional evaluation system that is easy to calculate from serum albumin, total cholesterol, and total lymphocyte count. This study showed that CONUT score is an independent scoring system that can predict outcomes in patients who received first-line chemotherapy for mCRC. We retrospectively assessed 211 consecutive mCRC patients receiving systemic first-line chemotherapy and found that CONUT score was significantly associated with PFS and OS. In addition, multivariate analysis indicated that CONUT score was an indicated prognostic factor for OS; higher CONUT score was significantly associated with worse prognoses. To our knowledge, this is the first study to show that CONUT score is an independent prognostic factor for $\mathrm{mCRC}$ patients receiving systemic chemotherapy.

CONUT score was first reported by Ignatio et al. (9), and is useful for evaluating the nutritional and immune status of patients. Additionally, CONUT score is a prognostic factor for patients with chronic diseases such as end-stage liver disease (16), heart failure (21), and several cancers $(23,24,25)$. Several previous reports have shown that CONUT scores are useful for estimating postoperative complications and prognosis in cases of esophageal cancer (12), gastric cancer (14) and intrahepatic cholangiocarcinoma (26). CONUT score has also proven to be a promising scoring system for predicting outcomes in CRC patients undergoing surgery $(15,23)$. However, there have not been any studies that assessed the relationship between CONUT score and mCRC patient prognoses.

Cancer progression has been shown to not only be affected by the malignant features of tumor cells themselves, but by the nutritional status of the patient (7). Furthermore, patient nutritional status has been associated with short- and longterm outcomes in CRC (5). Serum albumin concentration is one of the common nutritional indicators; however, albumin concentrations can be affected by patient status, such as liver function and body fluid volume. So, some reports have proposed adding plasma cholesterol levels to optimize the evaluation of nutritional status $(23,24)$. Hypocholesterolemia influences cell membrane fluidity, decreasing the mobility of cell surface receptors and their ability to transmit signals (27). Immune responses are dependent on lymphocytes; high numbers of infiltrating lymphocytes are associated with a good prognosis, whereas low lymphocyte levels are a recognized predictor of poor outcomes. CONUT scoring system includes plasma cholesterol levels, serum albumin and lymphocyte concentrations. Thus, CONUT score may be a good indicator of nutritional and inflammatory status.

Patient systemic inflammation and nutritional status may change during cancer progression (28). Several reports have shown that patient nutritional status is associated with shortand long-term outcomes in CRC $(23,27)$. Previously, Lu et al. reported the prognostic role of the platelet to lymphocyte ratio in CRC including mCRC (29). The platelet to lymphocyte ratio may reflect the patient's inflammatory and nutritional status. Kim et al. reported that a continuously high NLR or the change to a high NLR was also associated with poor OS and PFS in mCRC patients (28). The NLR is 

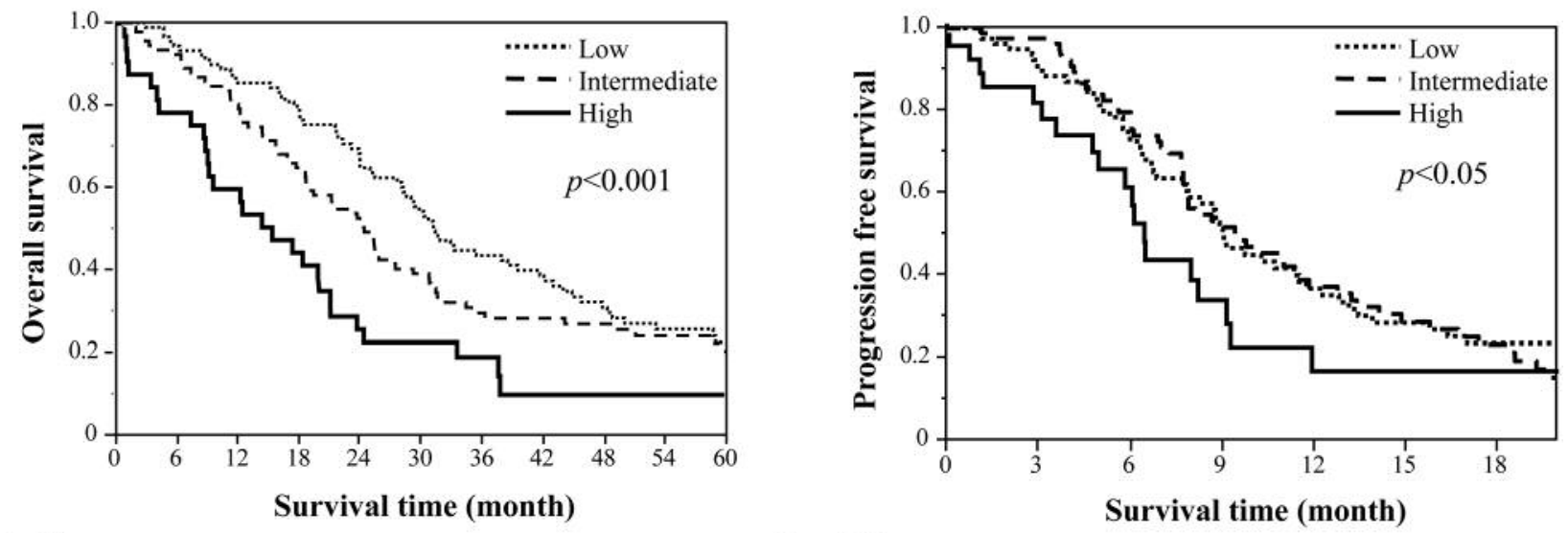

$\begin{array}{lrrrrrr}\text { No. at risk } & & & & & & \\ \text { Low } & 89 & 77 & 59 & 37 & 26 & 15 \\ \text { Intermediate } & 90 & 73 & 47 & 23 & 20 & 9 \\ \text { High } & 32 & 20 & 9 & 5 & 3 & 2\end{array}$

$\begin{array}{lr}\text { No. at risk } & \\ \text { Low } & 89 \\ \text { Intermediate } & 90 \\ \text { High } & 32\end{array}$

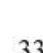

Figure 1. Overall survival and progression-free survival according to the CONUT score. Low: Low CONUT score (0-1) group; Intermediate: intermediate CONUT score (2-4) group; High: high CONUT score (5-) group.

Table IV. Univariate and multivariate analyses of prognostic factor for OS of this study.

\begin{tabular}{|c|c|c|c|c|c|c|}
\hline \multirow[t]{2}{*}{ Variables } & \multicolumn{3}{|c|}{ Univariate analyses } & \multicolumn{3}{|c|}{ Multivariate analyses } \\
\hline & HR & $95 \% \mathrm{CI}$ & $p$-Value & HR & $95 \% \mathrm{CI}$ & $p$-Value \\
\hline Age ( $\geq 75$ years) & 1.44 & $0.91-2.18$ & 0.11 & & & \\
\hline Gender (male) & 0.90 & $0.66-1.23$ & 0.51 & & & \\
\hline Body mass index $\left(<18.5 \mathrm{~kg} / \mathrm{m}^{2}\right)$ & 1.07 & $0.61-1.75$ & 0.80 & & & \\
\hline CEA $(>3.4 \mathrm{ng} / \mathrm{ml})$ & 1.44 & $0.96-2.24$ & 0.08 & & & \\
\hline CA19-9 (>37.0U/ml) & 1.45 & $1.08-1.97$ & $<0.05$ & 1.29 & $0.95-1.77$ & 0.10 \\
\hline Metastasis (synchronous) & 1.54 & $1.08-2.25$ & $<0.05$ & 1.55 & $1.02-2.43$ & $<0.05$ \\
\hline Metastatic lesions (multiple) & 1.57 & $1.16-2.14$ & $<0.05$ & 1.15 & $0.76-1.74$ & 0.51 \\
\hline Liver metastasis (not only) & 1.53 & $1.12-2.12$ & $<0.05$ & 1.38 & $0.88-2.18$ & 0.16 \\
\hline Primary Tumor location (right-side) & 1.94 & $1.40-2.68$ & $<0.001$ & 2.21 & $1.57-3.10$ & $<0.001$ \\
\hline Primary resection (no) & 1.56 & $1.15-2.13$ & $<0.05$ & 1.17 & $0.82-1.67$ & 0.40 \\
\hline KRAS status (wild type/mutant) & 1.15 & $0.77-1.76$ & 0.81 & & & \\
\hline CONUT score (High/Low+Intermediate) & 2.14 & $1.39-3.17$ & $<0.001$ & 2.01 & $1.26-3.12$ & $<0.05$ \\
\hline
\end{tabular}

OS: Overall survival; HR: hazard ratio; CI: confidence interval; CEA: carcinoembryonic antigen; CA19-9: carbohydrate antigen 19-9.

also a nutritional indicator. In this study, we assessed the prognosis of mCRC patients and their CONUT score. No previous studies have reported correlations between CONUT score and mCRC patient outcomes. Despite advances in therapy, mCRC still has a poor prognosis. Nagata et al. reported that mCRC patients with a high CONUT score had lower skeletal muscle mass (30). These patients had a shorter period of chemotherapy and lead to poor prognosis. Identifying patient status before chemotherapy could have several uses in clinical practice, including prognostic stratification and treatment aggressiveness. Early detection and the improvement of malnutrition may result in better patient outcomes.

This study had certain limitations; first, this was a retrospectively designed single-center study. Thus, the significance of CONUT score needs to be validated in other individual cohorts. Second, this study did not exclude several factors that may affect inflammation and nutritional status, such as non-steroidal anti-inflammatory drugs (NSAIDs) or lipid-lowering agents. However, we believe that these results will provide customized first-line therapy and improve mCRC patient outcomes. 


\section{Conclusion}

In conclusion, CONUT score is a useful prognostic marker for predicting long-term PFS and OS in mCRC patients.

\section{References}

1 Wang H, Naghavi M, Allen C, Barber RM, Bhutta ZA, Carter $A$ and Murray CJL: Global, regional, and national life expectancy, all-cause mortality, and cause-specific mortality for 249 causes of death, 1980-2015: a systematic analysis for the Global Burden of Disease Study 2015. The Lancet 388: 14591544,2016

2 Loupakis F, Cremolini C, Masi G, Lonardi S, Zagonel V, Salvatore L and Falcone A: Initial therapy with FOLFOXIRI and bevacizumab for metastatic colorectal cancer. N Engl J Med 371: 1609-1618, 2014.

3 Grothey A, Cutsem EV, Sobrero A, Siena S, Falcone A, Ychou $\mathrm{M}$ and Laurent D: Regorafenib monotherapy for previously treated metastatic colorectal cancer (CORRECT): an international, multicentre, randomised, placebo-controlled, phase 3 trial. The Lancet 381: 303-312, 2013.

4 Mayer RJ, Van Cutsem E, Falcone A, Yoshino T, GarciaCarbonero R, Mizunuma N and Group RS: Randomized trial of TAS-102 for refractory metastatic colorectal cancer. N Engl J Med 372: 1909-1919, 2015.

5 Barret M, Malka D, Aparicio T, Dalban C, Locher C, Sabate JM and Taieb J: Nutritional status affects treatment tolerability and survival in metastatic colorectal cancer patients: results of an AGEO prospective multicenter study. Oncology 81: 395-402, 2011.

6 Mohri Y, Inoue Y, Tanaka K, Hiro J, Uchida K and Kusunoki M: Prognostic nutritional index predicts postoperative outcome in colorectal cancer. World J Surg 37: 2688-2692, 2013.

7 Elinav E, Nowarski R, Thaiss CA, Hu B, Jin C and Flavell RA: Inflammation-induced cancer: crosstalk between tumours, immune cells and microorganisms. Nat Rev Cancer 13: 759-771, 2013.

8 Galdiero MR, Bonavita E, Barajon I, Garlanda C, Mantovani A and Jaillon S: Tumor associated macrophages and neutrophils in cancer. Immunobiology 218: 1402-1410, 2013.

9 Ignacio de Ulibarri J, Gonzalez-Madrono A, de Villar NG, Gonzalez P, Gonzalez B, Mancha A and Fernandez G: CONUT: a tool for controlling nutritional status. First validation in a hospital population. Nutr Hosp 20: 38-45, 2005.

10 Toiyama Y, Miki C, Inoue Y, Tanaka K, Mohri Y and Kusunoki M: Evaluation of an inflammation-based prognostic score for the identification of patients requiring postoperative adjuvant chemotherapy for stage II colorectal cancer. Exp Ther Med 2: 95-101, 2011.

11 Cabre M, Ferreiro C, Arus M, Roca M, Palomera E and SerraPrat M: Evaluation of CONUT for clinical malnutrition detection and short-term prognostic assessment in hospitalized elderly people. J Nutr Health Aging 19: 729-733, 2015.

12 Yoshida N, Baba Y, Shigaki H, Harada K, Iwatsuki M, Kurashige $\mathrm{J}$ and $\mathrm{Baba} \mathrm{H}$ : Preoperative nutritional assessment by Controlling Nutritional Status (CONUT) is useful to estimate postoperative morbidity after esophagectomy for esophageal cancer. World J Surg 40: 1910-1917, 2016.
13 Yoshida N, Harada K, Baba Y, Kosumi K, Iwatsuki M, Kinoshita $\mathrm{K}$ and Baba $\mathrm{H}$ : Preoperative controlling nutritional status (CONUT) is useful to estimate the prognosis after esophagectomy for esophageal cancer. Langenbecks Arch Surg 402: 333-341, 2017.

14 Kuroda D, Sawayama H, Kurashige J, Iwatsuki M, Eto T, Tokunaga $\mathrm{R}$ and Baba $\mathrm{H}$ : Controlling Nutritional Status (CONUT) score is a prognostic marker for gastric cancer patients after curative resection. Gastric Cancer 21: 204-212, 2017.

15 Tokunaga R, Sakamoto Y, Nakagawa S, Ohuchi M, Izumi D, Kosumi $\mathrm{K}$ and Baba $\mathrm{H}$ : CONUT: a novel independent predictive score for colorectal cancer patients undergoing potentially curative resection. Int J Colorectal Dis 32: 99-106, 2017.

16 Fukushima K, Ueno Y, Kawagishi N, Kondo Y, Inoue J, Kakazu $\mathrm{E}$ and Shimosegawa T: The nutritional index 'CONUT' is useful for predicting long-term prognosis of patients with end-stage liver diseases. Tohoku J Exp Med 224: 215-219, 2011.

17 Harimoto N, Yoshizumi T, Sakata K, Nagatsu A, Motomura T, Itoh $\mathrm{S}$ and Maehara Y: Prognostic significance of preoperative Controlling Nutritional Status (CONUT) score in patients undergoing hepatic resection for hepatocellular carcinoma. World J Surg 41: 2805-2812, 2017.

18 Takagi K, Yagi T, Umeda Y, Shinoura S, Yoshida R, Nobuoka D and Fujiwara T: Preoperative Controlling Nutritional Status (CONUT) score for assessment of prognosis following hepatectomy for hepatocellular carcinoma. World J Surg 41: 2353-2360, 2017.

19 Formiga F, Chivite D and Corbella X: Utility of the Controlling Nutritional Status (CONUT) score in patients admitted due to acute heart failure. Int J Cardiol 235: 203, 2017.

20 Shirakabe A, Hata N, Kobayashi N, Okazaki H, Matsushita M, Shibata Y and Shimizu W: The prognostic impact of malnutrition in patients with severely decompensated acute heart failure, as assessed using the Prognostic Nutritional Index (PNI) and Controlling Nutritional Status (CONUT) score. Heart Vessels 33: 134-144, 2017.

21 Nishi I, Seo Y, Hamada-Harimura Y, Sato K, Sai S, Yamamoto $\mathrm{M}$ and Ibaraki Cardiovascular Assessment Study-Heart Failure I: Nutritional screening based on the controlling nutritional status (CONUT) score at the time of admission is useful for long-term prognostic prediction in patients with heart failure requiring hospitalization. Heart Vessels 32: 1337-1349, 2017.

22 McShane LM, Altman DG, Sauerbrei W, Taube SE, Gion M, Clark GM and Statistics Subcommittee of the NCIEWGoCD: REporting recommendations for tumour MARKer prognostic studies (REMARK). Br J Cancer 93: 387-391, 2005.

23 Iseki Y, Shibutani M, Maeda K, Nagahara H, Ohtani H, Sugano $\mathrm{K}$ and Hirakawa $\mathrm{K}$ : Impact of the preoperative Controlling Nutritional Status (CONUT) score on the survival after curative surgery for colorectal cancer. PLoS One 10: e0132488, 2015.

24 Toyokawa T, Kubo N, Tamura T, Sakurai K, Amano R, Tanaka $\mathrm{H}$ and Ohira M: The pretreatment Controlling Nutritional Status (CONUT) score is an independent prognostic factor in patients with resectable thoracic esophageal squamous cell carcinoma: results from a retrospective study. BMC Cancer 16: 722, 2016.

25 Akamine T, Toyokawa G, Matsubara T, Kozuma Y, Haratake N, Takamori S and Maehara Y: Significance of the preoperative CONUT score in predicting postoperative disease-free and overall survival in patients with lung adenocarcinoma with obstructive lung disease. Anticancer Res 37: 2735-2742, 2017. 
26 Miyata T, Yamashita YI, Higashi T, Taki K, Izumi D, Kosumi K and Baba H: The Prognostic impact of Controlling Nutritional Status (CONUT) in intrahepatic cholangiocarcinoma following curative hepatectomy: A retrospective single institution study. World J Surg 42: 1085-1091, 2017.

27 Mamtani R, Lewis JD, Scott FI, Ahmad T, Goldberg DS, Datta $\mathrm{J}$ and Boursi B: Disentangling the association between statins, cholesterol, and colorectal cancer: a nested case-control study. PLoS Med 13: e1002007, 2016.

28 Chua W, Charles KA, Baracos VE and Clarke SJ: Neutrophil/lymphocyte ratio predicts chemotherapy outcomes in patients with advanced colorectal cancer. Br J Cancer 104: 1288$1295,2011$.
29 Lu C, Gao P, Yang Y, Chen X, Wang L, Yu D and Wang Z: Prognostic evaluation of platelet to lymphocyte ratio in patients with colorectal cancer. Oncotarget 8: 86287-86295, 2017.

30 Nagata T, Nakase Y, Nakamura K, Sougawa A, Mochiduki S, Kitai $S$ and Inaba S: Prognostic impact of a nutritional index including muscle volume in stage 4 colorectal cancer. In Vivo 30: 885-892, 2016.
Received June 9, 2018

Revised June 26, 2018

Accepted July 11, 2018 